\title{
INTELLIGENT MULTI-AGENT FUZZY CONTROL SYSTEM UNDER UNCERTAINTY
}

\author{
Ben Khayut ${ }^{1}$, Lina Fabri ${ }^{2}$ and Maya Abukhana ${ }^{3}$ \\ ${ }^{1}$ Department of R\&D, IDTS at Intelligence Decisions Technologies Systems, \\ Ashdod, Israel \\ ben_hi@hotmail.com \\ ${ }^{2}$ Department of R\&D, IDTS at Intelligence Decisions Technologies Systems, \\ Ashdod, Israel \\ lina.fabri@gmail.com \\ ${ }^{3}$ Department of R\&D, IDTS at Intelligence Decisions Technologies Systems, \\ Ashdod, Israel \\ maya.kh111@gmail.com
}

\begin{abstract}
The traditional control systems are a set of hardware and software infrastructure domain and qualified personnel to facilitate the functions of analysis, planning, decision-making, management and coordination of business processes. Human interaction with the components of these systems is done using a specified in advance script dialogue "menu", mainly based on human intellect and unproductive use of navigation. This approach doesn't lead to making qualitative decision and effective control, where the situations and processes cannot be structured in advance. Any dynamic changes in the controlled business process make it necessary to modify the script dialogue. This circumstance leads to a redesign of the components of the entire control system. In the autonomous Fuzzy Control System, where the situations are unknown in advance, fuzzy structured and artificial intelligence is crucial, the redesign described above is impossible. To solve this problem, we propose the data, information and knowledge based technology of creation Situational, Intelligent Multi-agent Control System, which interacts with users and/ or agent systems in natural and other languages, utilizing the principles of Situational Control and Fuzzy Logic theories, Artificial Intelligence, Linguistics, Knowledge Base technologies and others. The proposed technology is defined by a) methods of situational fuzzy control of data, information and knowledge, b) modelling of fuzzy logic inference, c) generalization and explanation of knowledge, d) fuzzy dialogue control, e) machine translation, f) fuzzy decision-making, g) planning and $h$ ) fuzzy control of organizational unit in real-time under uncertainty, fuzzy conditions, heterogeneous domains, multi-lingual communication in Fuzzy Environment.
\end{abstract}

\section{KEYWORDS}

Intelligent fuzzy control

David C. Wyld et al. (Eds) : CST, ITCS, JSE, SIP, ARIA, DMS - 2014 


\section{INTRODUCTION}

\subsection{Analysis}

Data, information and knowledge are one of the main components in the world development. Their correct use leads to the adoption of relevant decisions and effective control in Intelligent Multi-agent Fuzzy ControlSystem (IMAFCS).

Traditional Control Systems are using a "Menu" principle to interact with their users (analysts, experts, managers). Each professional within its subject area, spends an unproductive time to navigate the menu dialog instead of solving their day to day tasks. They can be more productive in interacting with the applications in a more natural and less predefined way, where the system itself should find the required and relevant data, information or knowledge to support their tasks. Changes in business process of organizational unit entail changes in the script menu dialog. This leads to a permanent and continuous redesign of the Control system. In these circumstances, since the technology doesn't support the business process need, it is impossible to make relevant realtime decisions and control. To avoid such a discrepancy it becomes necessary to use the principle of Situational Control [6]in the design of the Intelligent Control System (ICS). Given the uncertainty of the environment, as well as increased complexity of business processes and technologies, the use of Fuzzy Logic [1] and Artificial Intelligence becomes necessary. A multilingual LinguisticProcessor (LP) should be used to support human-machine interaction to incorporate a business process into the control system. For real time decision making and fuzzy control in a fuzzy environment we suggest to use of Fuzzy Logic Inference [13], Generalization and Explanation of knowledge [15], Dialog Control [11] and other methods. Obviously, the considered IMAFCS should be able to support a variety of subject areas. The solution of these tasks in this article is focused on the systematic approach of modeling, planning and controlling of linguistic and subject area data, information, knowledge, fuzzy logic inference and others, by mapping the objectives and constraints in fuzzy environment.

The novelty of the technology which designs IMAFCS consists of:

- Modelling and situational fuzzy control of data, information and knowledge for implementing an automatic fuzzy inference and finding a correct, accurate, timely and adequate decision, taking into account a current situation and impact of fuzzy environment.

- Using of resulting decision, criteria and purpose for providing of modelling, planning and control of the business process in the fuzzy environment.

- Converting and deriving images, concepts, meanings from natural languages in various subject areas and serializing them into the bases of data, information and knowledge.

- Use of these bases for multi-lingual human - machine communication using methods of dialog control, generalization and explanation of knowledge in the intelligent fuzzy control system.

- Use of properties of a) atomicity of data, b) relationality of information, c) figurativeness of knowledge for their integration and aggregation.

- Using methods of wisdom, intuition and behavior and others to obtain decision of high quality and precision.

The analysis of the state of scientific research in the field of design intelligent control systems showed that the directions and methods of implementation are related mainly to their functionality. In this context, we will hold a brief of comparative analysis of the functionality of the IMAFCS, offered by us and other authors. 
In [12] is given an interesting overview of the approach to the problem of Fuzzy Control. Our approach differs from the mentioned, the fact that in addition to proposed methods of formalization we taking into account the principles of situational control, artificial intelligence and others. This allows realizing fuzzy control in situation, which unknown in advance. At the same time, we have developed modelling techniques [16] based on the managed data, information and knowledge [10] that allows finding relevant solution with the desired accuracy in the circumstances. This accuracy is implemented using intelligent agents of analysis, decisionmaking, planning and others by using the values of fuzzy membership function.

In [17] is represented linguistic approach for solving decision problems under linguistic information using Multi-criteria decision making, linguistic modeling, aggregation and linguistic choice functions methods on base of rank ordering among of the alternatives for choosing the best of them.

The main difference between our systematic approach and the proposal is a:

- Generalized notion of linguistic variable of Fuzzy Logic, by which we evaluate and take into account not only the morphological, syntactic and semantic, but also, behavioural, psychological and other aspects of the terms (atomic units) of Natural Language (NL).

- Situational Fuzzy Control in Fuzzy environment, by which we control not only information, but also data, knowledge, decisions, agents and others.

- Decision-making process is based not only on using the rank for estimation of the alternatives, but also on automatic Fuzzy Logic Inference, Planning, Control of alternatives, situations and other units.

- Multi-lingual interaction, generalization, explanation, serialization, storage and actualization of knowledge in fuzzy conditions, heterogeneous subject areas, where the situations are unknown in advance, fuzzy structured and not clearly regulated.

In [19] are considered adjustable autonomous agents that possess partial knowledge about the environment. In a complex environment and unpredictable situations these agents are asked the help of human on base of the model, called HHP-MDP (Human Help Provider MDP) and requests, which are set in advance.

The comparative analysis of these and other works, associated with our work, showed, that there is no integrated, systematic and linguistic approach to the problem of situational fuzzy control in a fuzzy environment, including the techniques of situational control of fuzzy data, information and knowledge, modeling, planning, decision-making, dialog control and situational fuzzy control of the organizational unit, based on the achievements of Fuzzy Logic, Situational Control, Artificial Intelligence, Linguistics and others.

In this article, we present the results of our studies and the approach to the design of IMAFCS using our developed methods and tools.

\subsection{Terminology}

Data is organized in the memory and are perceived by the person or machine as facts, numbers, words, symbols, lines and other items of information. They are not related to each other and are found intexts, pictures and othermaps of reality.

Information is a group of related data, organized in the memory that respond to the questions of "who", "what", "where", "when" and others. 
Knowledge is the image or domain model, extracted from information and organized in memory, which in itself are interpreted, structured, linked, associated, transformed, compared, upgraded, activated, analyzed, deduced, built, serialized and so on, in real time. The mentioned image or domain should respond to questions "why" and "how", consider the impact of environment and specificity of subject area, satisfying the criteria and purpose of existence.

Wisdom is a method of perceiving reality and achieving a unique solution (answer) on the basis of intelligence, archival knowledge (experience), principles and inference in a certain situation.

Intuition is a method of perceiving reality and a achieving a unique solution (answer) on the basis of intelligence, archival knowledge (experience) principles and unique inference in an extreme situation.

Modelling decisions is defined as construction of a new conceptual situation and a state of controlled units (fuzzy data, information, knowledge, inference and others), which meets the criteria and purposes of the information system in fuzzy environment. The purposes are functions of the information system.

Planning decisions is defined as a use of modelling results to create a sequence of alternative decisions that will match to the situation and the state of information system in the subsequent stages of management of the organizational unit.

Decision-making is defined as a process of modelling fuzzy logic inference [13] for selection the relevant decision from limited number of alternative decisions, obtained during the planning decisions.

Fuzzy Control is the process of using the modelling results of planning and decision-making in fuzzy environment, in order to implement a control action on the units (data, information, knowledge, decisions, organizational unit and others) to shift them and their control system to a new state, that matches a specified criterion.

Under the fuzzy logic inference [13] we mean procedure for determining the vectorof internal and external output fuzzy variables $b_{k}^{i} \in V_{k}^{m}$ using a newvector of the values of the inputfuzzyvariables $a_{k}^{i} \in U_{k}^{m}$, whichtransforms the IMAFCS in its new state. This procedure is implemented on the extensional, intentional and reformativelevels of modelling knowledge [16].

Under the DialogControl we means the process of presenting partners of common commands(questions) to each other and providing by them targeted actions (issuing replies) relevant to the subject of the dialogue and the situations in which itoccurs.

Intelligent fuzzy control system is understood as a knowledge based system, which is reliably electronic autonomous system, and which a) operates at a high-level operating system b) connected to the Internet, d) executes a native or cloud-based applications, e) analyzes the collected data, information and knowledge, and e) realizes the human-machine functions for solving problems in fuzzy environment.

Traditional Control System is an Information System, which is working on base of "menu" scenarios and is not autonomous.

According Wikipedia the Organizational Unit $O^{U}$ (Figure 2, Figure 3) represents a single organization with multiple units (departments) within that organization. 
The business process is an activity or set of activities in organizational unit $O^{U}$ that will realizes a specific organizational goal.

Subject area understood by us as branch of knowledge and technologies, where the organizational units are functioning.

Environment is the surrounding reality, consisting of organizational units, information systems, robots, agents, agent systems and so on, which interact with each other under the influence of the environment.

A multi-agent system (MAS) [18] is a computational system where agents cooperate or compete with others to achieve some individual or collective task.

Agent is a real-world or artificial entity, which is a person (in the first case) and an object (in the second case), and which are capable of performing some action or service or otherwise, interacting with other entities.

Figure 1 depicts nesting of the above-defined concepts.

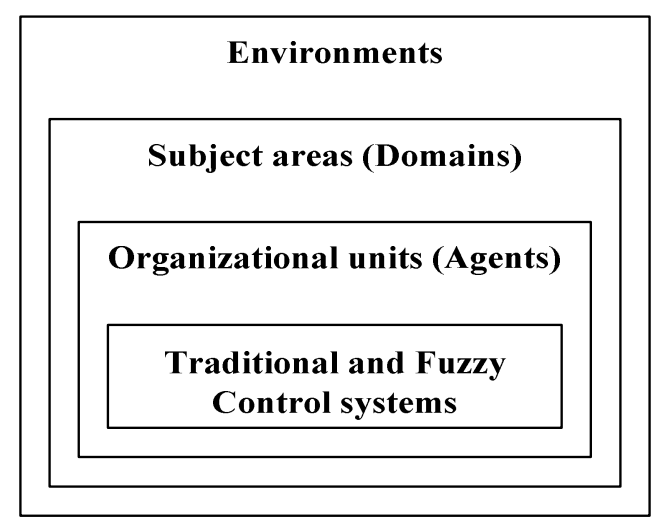

Figure 1. Control systems and environments

Thus, the above defined and implemented in the computer concepts are agents.

Combining, nesting and integrating the agents into the groups according to their objectives and functional features turn them into an IntelligentMulti-agent System in the paper.

Fuzzy Control provides a formal methodology for representing, manipulating, and implementing a human's heuristic knowledge about how to control a system [12].

According Wikipedia the Fuzzy Control System is a control system based on fuzzy logic - a mathematical system that analyzes analog input values in terms of logical variables that take on conditions values between 0 and 1 , in contrast to classical or digital logic, which operates on discrete values of either 1 or 0 (true or false, respectively). 


\section{CONTENT}

\subsection{Conception}

The main purpose of the IMAFCS function is to facilitate the analysis, planning, decision-making, fuzzy control and coordination of organizational unit on the basis of the intended target and the current situation. Under the facilitating in IMAFCS is understood the full use of intelligence of the system to perform these functions by interacting with it in natural language. In this connection the operation of the system should be based on the perception, processing and synthesizing knowledge in real time.

Given the fact that these processes occur in unexpected situations and Fuzzy Environment(people, robots, nature, space, hardware, software, other information and agent systems, and so on) becomes necessary to use the theories Situational Control[6], Fuzzy Logic [1] and other methods.

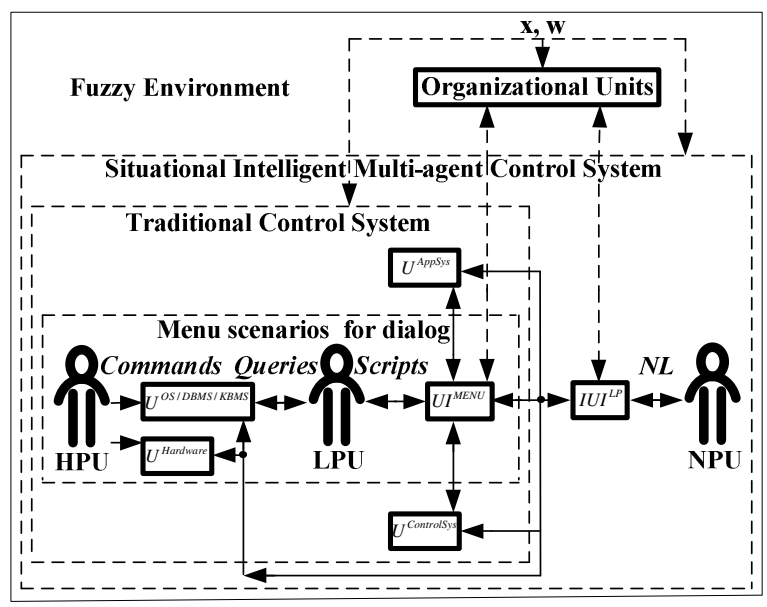

Figure 2.Human Interaction in Situational, Intelligent Multi-agent and Traditional Control Systems.

The paper proposes the creation technology of the IMAFCS that integrates the achievements of Situational Control, Fuzzy Logic, Linguistics, Artificial Intelligence and others for realization of the following functionalities:

- To use of models of representation of linguistic andsubject area data, information and knowledge in FuzzyEnvironment.

- Situationalfuzzy Modelling, Decision-making, Control and Planning in conditions of the absence, incompleteness, vagueness and ambiguity of knowledge.

- FuzzyLogic Inference.

- Generalization and Explanation of knowledge.

- Fuzzy Dialog Control.

- Linguistic Processor and Multi-lingualinteraction in Fuzzy Environment.

The main features of the proposed technology of design IMAFCS lies in the use of methods:

- Situational control of data, information and knowledge and implementation by it of automatic processes of inference, making the right decisions, generalization and explanation knowledge, dialog control, planning and management of organizational unit. 
- Creation $L P$, which is a part of the Intelligent Interface and enabling people to interact with him in Natural Language without "menu" dialog.

The group of highly professional users (HPU) (Figure 2) includes users, which were trained in the use and maintenance of hardware, software, networks, data, information and knowledge bases.

A group of less professionalusers (LPU) in (the mentioned above resources)are developers: systemanalysts, application programmers, testers, operators and others.

The group of not professionalusers (NPU) is composed of experts in their field, who use the functionality of information system to solving their functional tasks. This group includes the decisionmakers, analysts, experts, consultants, managers and other experts in their subject area.

$U I^{M E N U}$ is the user interface of dialogue menu in Traditional Information System, with whom interacts user LPU using script dialogue language. This useralsointeracts with interfaces $U^{O S / D B M S / K B M S}$, using the query language.

$U^{\text {OS IDBMS / KBMS }}$ aregroup of interfaces, used by users HPU for system support the Operation Systems (OS), Data Base Management Systems (DBMS) and Knowledge Base Management Systems using commands languages

$U^{\text {AppSys }}, U^{\text {Controlsys }}$ are respectively, Application and Control systems, which realize the functionalities of the considered SIIS and other systems in it.

$U I^{L P}$ isintellectual interface with built in LP and with whom interacts the user NPU using natural language. This interface is connected with interfaces $U^{O S / D B M S / K B M S}, U I^{M E N U}$ and with systems $U^{\text {AppSys }}, U^{\text {Controlsys }}$ for the use of the existing functionalities of other information systems. Thus, IMAFCSintegrates the functionality of existing traditional control systems to solve simple problems and its intellectual capabilities to solve complex problems in fuzzy environment.

The $U^{\text {Hardware }}$ ishardware resources, supported by user HPU.

The interface $I U I^{L P}$ interacts with the organizational entities, performing all functionality of ICS by returning to user NPU the results in the required form. Similarwork is done by the user LPU, which interacts with the organizational units through theinterface $U I^{M E N U}$.

Intellectualization of labor of the LPU and HPU users is not considered in this paper and will be the subject of further study. These studies involve the introduction of artificial intelligence in hardware, software and networks, and the inclusion of these groups in the NPU group.

\subsection{Methods}

\subsubsection{The models and methods of representation of linguistic and subject data, information and knowledge in Fuzzy Environment.}

In [13], [10]were extended the concept of a linguistic variable, formal and semiotic models with using the principle and method of situational control [6] by taking into account of the accepted 
methods of representation, organization, integration, processing and synthesis of data, information and knowledge [3-5], [7,8], [14]. Through the use of fuzzy sets theory and situational control model were defined linguistic and thematic units, attributes, corteges and dictionary entriesin linguistic and thematic relational bases of data, information and knowledge.

These models definea conceptual means of presenting and structuring of data, information, knowledge in fuzzy environment, and are also used for modeling, planning, decision-making and control in ICS.

Thus, the intellectuality of the Data, Information and KnowledgeControl System consists in providing of interaction of decision-maker with consultants and experts (last among themselves) in order to organize dialogue between them in a natural language.

\subsubsection{Situational Fuzzy Modelling, Decision-making, Control and Planning in conditions of the absence, incompleteness, vagueness and ambiguity of knowledge.}

In order to control an organizational unit it is required to now its structure, the purpose of its existence and its control criteria [6].

The task becomes more complicated when there is a need to control organizational units in real time, in situations unexpected in advance, using variety of natural languages and subject areas.

In these circumstances, arises a problem of decision making in fuzzy environment [2] based on the data, information and knowledge.

The solution to this problem implemented by a) methods of modelling, planning and controlling of linguistic and subject area data, information, knowledge, fuzzy inference and others, b) mapping the objectives and constraints in fuzzy environment $[16,13]$.

\subsubsection{Fuzzy Inference}

Given the complex character of functioning of the ICS, its design is impossible without the use of theories situational control [6], fuzzy sets [1] and the proposed above of models of representation, synthesis, modelling, planning and management of data, information and knowledge.

Therefore, the modeling method of fuzzy inference can be applied to data control system, satisfying the following principles [13], [10]:

- All information about the data, information and knowledge (about the organizational unit) may be communicated to the control system as a set of phrases of Natural Language.

- Control model is fundamentally should be open and never ends the creation of the final formal model.

- Description of the data management (information, knowledge) process is possible in the form of natural phrases and $\backslash$ or another language.

In these circumstances, the proposed modeling method of fuzzy inference is implemented by a system of situational data controland displays alinguistic approach to the problem. The method allows realizing the inductive and deductive inferencein natural language in integrated subject areas, based on incoming fuzzyfragments (parcels) of the language.

To do this, we used the heuristic algorithms, methods of wisdom, intuition, behaviour and other algorithms and methods that invoke the modules of modelling of data, information and knowledge. The algorithms and methods uses generalized linguistic variables, fuzzy sets, rules 
and facts (situations), previous decisions and their subsets (segments), extracted from bases of data, information and knowledge to obtain relevant decision.

The detailed presentation of the fuzzy logic inference is given in [13].

\subsubsection{Generalization and Explanation of knowledge}

The task of generalization of knowledge is reduced to finding the target (unique) situation $Q_{l}$ of data, information and knowledge by using of their current situation $Q_{j}$ and process of control them on base of model [15].

The decision, correspond to the found situation of the data, information and knowledge, shifts them from the current situation $Q_{j}$ into a new $Q_{l}$.

This decision (action) determines the impact rulesI on data (information and knowledge), which must be met in the overall situation $S_{i}$, so that they and control system would correspond to the new (changed) situation $Q_{l}$.

The target function in the model of generalization and explanation of knowledge defines the purpose of control of data, information and knowledge. The purpose may be the stirring up of processes of modelling, decision-making, planning, control, generalization and explanation knowledge and other.

\subsubsection{Dialog Control}

In this paper, we propose an approach to the control of the dialogue [11]in ICS using the modelling and controldata, information and knowledge [10], fuzzy inference [13], generalization and explanation knowledge [15] and others.

Together with the secontrol systems, Dialogueis controlled by planningsystem, which uses the model, created by interpreter of dialogue $U^{D}$ (Figure 3) and sub systems of fuzzy inference $U^{I}$, generation and explanation of knowledge $U^{G}$.Those sub systems provide processing and forming the input and output messages of natural language in knowledge management system usingbases of subject area and linguistic data, information and knowledge.

The $I U I^{L}$ is a Intelligent User Interface (IUI) with the inclusion of LP. Together with the Manager the $I U I^{L}, U^{D}, U^{I}, U^{D}$ are representing the Decision-making system. The $U^{A}$ (analyst, reviewer) and $U^{E}$ (expert, approver) are intelligent subsystems, which are support the decision-making process. 


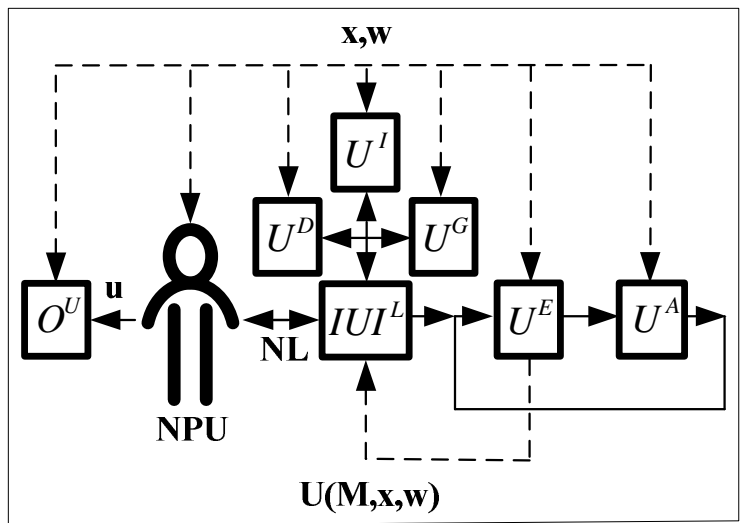

Figure 3. Dialog Control in human interaction.

\subsubsection{Linguistic Processor and Multi-lingual interaction in Fuzzy Environment}

The users interact with IUI using natural language, which is processed by LP. It is represented by the Interpreter $(D)$ andSynthesizer $(R)$ (Figure 2).

Its implementation involves the functionality $[3,5]$ of:

- Providing meaningful machine translation for identification concepts.

- The adequacy of the mapping meanings of the concepts expressed by the termin a particular language in the context ofa particular subject area.

- Opportunity to endowa specific term by attributes of grammar, logic, semantics, pragmatics, psychology and others in accordance with its meaning in a particular subject area and the context ofuse.

- Implementation of the "understanding" by means the algorithms of synthesis output expressions of natural language based on logical-semantic characteristics of intralinguistic representations of meaning.

- Resolution disambiguation expressions used languages by sampling lexical and semantic characteristics and vocabularyof languages and conducton the basis of theirlexical and semantic analysis to determine these manticmatching of input and output language equivalents.

- The possibility of implementing a system of automaticmachinedictionariesandof thesauriin linguisticdatabaseswithconceptual connectionto the subjectdatabases and knowledge.

Implementation of Machine Translation terminological phrases includes the blocks of:

- Logical-semantic, grammatical analysisof input combinations and the identification of concepts of its outputequivalents.

- Prior authorization of lexicalambiguityon inputphrasesusing conceptual codes.

- Extraction of grammatical information.

- Grammatical ambiguity resolutionbased on the stems of input combinations.

- Extracting logical information.

- Final resolution of lexicalambiguity.

- Resolution of lexical and grammatical ambiguity of input and output combinations of stems.

- Logical and semantic disambiguation of input and output terminological expressions.

- Identification of concepts and the formation of natural language expressions of output terminological phrases. 
The LP realizes transformation target's actions, which are expressed in NL and other languages. The method of realization of the LP is represented in [3].

\section{CONCLUSIONS}

The proposed methods and technology are oriented for design and development of autonomousSmart/ Intelligent Multi-agent Fuzzy Control System, which will be operated in fuzzy environment, interacting with people and other systems and agents in different languages and dissimilar subject areas, where the situations and factors of influence on the control unit cannot be determined and structured in advance.

A distinctive feature of this approach is to target the modelling and control of fuzzy linguistic and subject data, information, knowledge, alternative solutions, objectives and constraints in order to find accurate, relevant and right decisions, which are suitable to the situation, with regard to external and internal influences of the fuzzy environments on the system.

The results of this work focused on the creation of autonomous, situational, intelligent, multiagent informationcontrol systems of robots, unmanned production and of Apparatus, functioning in the fuzzy environment and unforeseen situations in advance.

\section{REFERENCES}

[1] L. A. Zadeh, (1970) "Fuzzy Sets", Information and Control, 8, New York, pp. 338-359.

[2] R. E. Belman\& L. A. Zadeh, (1970) "Decision-making in fuzzy environment", Management Sciences, California, vol. 14, pp. 141-164.

[3] B. Z. Khayut, G. I. Nikolaev, A. N. Popeskul, V. A. Chigakovsky, (1983 ) "Realization Linguistic Data Base for Machine translation foreign languages texts using DBMS INES", Collection of papers, International Machine Translation Symposium, Moscow, pp. 236-237.

[4] B. Z. Khayut\& G. I. Nikolaev, (1984 )"Data organization for interactive systems "understanding" of natural language and multilingual interaction", Workshop, Interactive systems and their practical application, Chisinau, pp. 165-166.

[5] G. I. Nikolaev\& B. Z. Khayut, (1985 )"Logical-semantic method of identifying noun phrases of natural language”, Workshop, Logic and combinatorial methods in artificial intelligence and pattern recognition, Chisinau, pp. 79-80.

[6] D. A. Pospelov, (1986 )“Situational Control”, Science, Moscow, 288 pages.

[7] B. Z. Khayut, (1986)"An approach to the integration of heterogeneous databases in design of intelligent access", Workshop, Interactive systems and their practical application, Russia, Kalinin, pp. $164-165$.

[8] B. Z. Khayut, (1986) "The method of constructing an integrated system of intelligent access and data control”, Workshop, Methods for building integrated software systems of data control, Russia, Kalinin, pp. 179-180.

[9] G. I. Nikolaev, Y. N. Pechersky, B. Z. Khayut, (1986) "The conceptial approach to the presentation and synthesis of knowledge about natural language and subject area for the "man-intelligent robots", Workshop, Automation and robotizing of manufacture with the use of microprocessor-based producrs, Chisinau, pp. 67-68.

[10] B. Z. Khayut\& Y. N. Pechersky, (1987) "Situational Data Control", VINITI, Deposited manuscript, Moscow, 29 pages.

[11] Y. N. Pechersky\& B. Z. Khayut, (1988)"Approach to Dialog Control in the situational decisionmaking system”, Workshop, Interactive tools in automated control systems, Chisinau, pp. 169-170.

[12] K. M. Passino\& S. Yurkovich, (1988 ) "Fuzzy Control”, Ohio State University, Addison-Wesley, Menlo Park, California, pages 502.

[13] B. Z. Khayut, (1989 )"Modelling of fuzzy logic inference in the decision making system", Science, Issue 110, Academy of Sciences of Moldova, Chisinau, pp. 134-144. 
[14] B. Z. Khayut\& Y. N. Pechersky, (1989) "Knowledge modelling in decision support system" VINITI, Deposited manuscript, Moscow, 12 pages.

[15] Y. N. Pechersky\&B. Z. Khayut, (1989 )“Generalization of knowledge in decision support system”, Workshop, Questions of development computer facilities, Chisinau, pp. 107-108.

[16] B. Z. Khayut, (2000) "The method of modelling and decision-making using fuzzy data", fourth collection of scientific papers, Creative searches of scientific Israel today, Ashkelon, pp. 130-133.

[17] F. Herrera\& E. Herrera-Viedma, (2000) "Linguistic decision analysis: steps for solving decision problems under linguistic information”,Elsevior Science, Fuzzy sets and systems, vol. 115, Issue 1, pp. 67-82.

[18] J. W. Krupansky, (2005 )“Advancing the Science of Software Agent Technology”, Agtivity.

[19] N. Cote, A. Canu, M. Boused, A, Mouaddib, (2012) "Humans-Robots Sliding Collaboration Control in Complex Environments with Adjustable Autonomy", Web Intelligence and Intelligent Agent Technology (WI-IAT), IEEE/WIC/ACM International Conferences, vol. 2, pp. 146-153.

\section{AUTHORS}

Ben Khayut graduated MSc in Mathematics at the University of Chisinau and PhD in Mathematical Cybernetics (completed studies) at the Institute of Mathematics of the Academy of Sciences of the Moldova in the field of applied mathematics, computer science, artificial intelligence, fuzzy logic, where he defended the pre-thesis "Data Control in decision-making on natural language". Has experience (over 30 years) in design and development of Information Control systems in industry using linguistic and subject area data, information and knowledge bases, and algorithms of machine translation as researcher, designer, programmer, project manager, using modern scientific methods, software and hardware. Have 25 scientific papers. In 2007 he founded the Intelligence Decisions Technologies Systems (IDTS).

Lina Fabri graduated Bachelor, Economics and Management at the College of Business Management. Have 14 years of successfully leading and executing Business Intelligence and Technology strategies and solutions to drive business value. Proven ability to define and drive technology solutions and services with solid track record of high performing standards and excellence. Has good scientific ability and experience in $R \& D$ of Information Control Knowledge based Systems, Planning and Decision-making using business strategy methods in various subject areas using methods of Situational Control, Linguistics and Artificial Intelligence.

Maya Abukhana graduated Information systems and Management at the College of Business Management. Have 9 Years of successfully leading and executing Business Projects of Financial and Trading systems to drive business value. Proven ability to define and drive technology solutions and services with solid track record of high performing standards and excellence. Has a good scientific ability and experience in R\&D in the design of knowledge bases, architecture and algorithms for complex financial control systems, as well methods of human interaction using Linguistics and Database technologies.
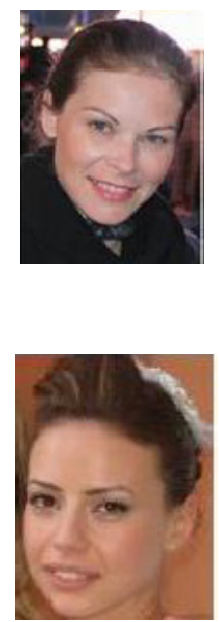